PROCEEDINGS OF THE

AMERICAN MATHEMATICAL SOCIETY

Volume 133, Number 11, Pages 3323-3331

S 0002-9939(05)07894-9

Article electronically published on May 9, 2005

\title{
SINGULAR PERTURBATION PROBLEMS FOR TIME-REVERSIBLE SYSTEMS
}

\author{
CLAUDIO A. BUZZI, PAULO RICARDO DA SILVA, AND MARCO ANTONIO TEIXEIRA \\ (Communicated by Carmen C. Chicone)
}

\begin{abstract}
In this paper singularly perturbed reversible vector fields defined in $R^{n}$ without normal hyperbolicity conditions are discussed. The main results give conditions for the existence of infinitely many periodic orbits and heteroclinic cycles converging to singular orbits with respect to the Hausdorff distance.
\end{abstract}

\section{INTRODUCTION}

The present work fits within the geometric study of singular perturbation problems expressed by one-parameter families of vector fields on $R^{m+n}$. Special emphasis on systems having some symmetry properties is given. A singular perturbation problem is expressed by a differential equation $z^{\prime}=h(z, \varepsilon)$ with $z \in R^{n+m}, \varepsilon \geq 0$ and $h \in C^{\infty}$, where we want to study the phase portrait for sufficient small $\varepsilon>0$ near a set that contains a manifold of singular points of $z^{\prime}=h(z, 0)$.

Let $z=(x, y) \in R^{n+m}$ and $f, g$ be smooth functions. We deal with equations that may be written in the form

$$
\left\{\begin{array}{l}
x^{\prime}=f(x, y, \varepsilon), \\
y^{\prime}=\varepsilon g(x, y, \varepsilon),
\end{array} \quad x=x(\tau), y=y(\tau) .\right.
$$

The main trick in the geometric singular perturbation (GSP) is to consider the family (1.1) in addition to the family

$$
\left\{\begin{array}{c}
\varepsilon \dot{x}=f(x, y, \varepsilon), \quad x=x(t), y=y(t) \\
\dot{y}=g(x, y, \varepsilon),
\end{array}\right.
$$

obtained after the time rescaling $t=\epsilon \tau$.

Equation (1.1) is called the fast system and (1.2) the slow system. Observe that for $\varepsilon>0$ the phase portrait of fast and slow systems coincide.

For $\varepsilon=0$, let $\mathcal{S}$ be the set of all singular points of 1.1. . We call $\mathcal{S}$ the slow manifold of the singular perturbation problem, and it is important to note that equation (1.2) defines a dynamical system, on $\mathcal{S}$, called the reduced problem.

Combining results on the dynamics of these two limiting problems (1.1) and (1.2), with $\varepsilon=0$, one obtains information on the dynamics for small values of $\varepsilon$. In

Received by the editors March 4, 2004 and, in revised form, June 21, 2004.

2000 Mathematics Subject Classification. Primary 34C14, 34C20, 34D15.

Key words and phrases. Singular perturbations, time-reversible systems.

The first author was partially supported by CAPES 0092/01-0.

The second author was partially supported by CAPES 0092/01-0 and CNPq 476886/2001-5. 
fact, such techniques can be exploited to formally construct approximate solutions on pieces of curves that satisfy some limiting version of the original equation as $\varepsilon$ goes to zero.

Definition 1.1. Let $\mathcal{N}_{1}$ and $\mathcal{N}_{2}$ be normally hyperbolic invariant manifolds on $\mathcal{S}$ for the reduced problem. A singular orbit consists of 3 pieces of smooth curves: an orbit of the reduced problem in the unstable manifold $W_{1}^{u}\left(\mathcal{N}_{1}\right)$, an orbit of the reduced problem in the stable manifold $W_{2}^{s}\left(\mathcal{N}_{2}\right)$ and a heteroclinic orbit of the fast problem connecting the two previous pieces.

Definition 1.2. Let $A, B \subset R^{n+m}$ be compact sets. The Hausdorff distance between $A$ and $B$ is $D(A, B)=\max _{z_{1} \in A, z_{2} \in B}\left\{d\left(z_{1}, B\right), d\left(z_{2}, A\right)\right\}$.

The main question in GSP-theory is to exhibit conditions under which a singular orbit can be approached by regular orbits for $\varepsilon \downarrow 0$, with respect to the Hausdorff distance.

The usual approach is to consider a smooth manifold of singular points along which the vector field $h(z, 0)$ is normally hyperbolic. We refer to [2] for a survey of GSP-theory and related problems. A general question is what remains of this picture when the normal hyperbolicity assumption is dropped. That means there exists a turning point in the usual terminology. In the generic case, a perturbation problem is not expected to have an easy answer. We deal with such a problem by assuming that our system is time-reversible (see [1], [3], [6] and [7] for such problems in different contexts). It is worthwhile to mention that the reversibility will guarantee that the center manifold is symmetric with respect to the fixed points set of the involution. So, if we assume that the turning point belongs to this fixed set, then it can be treated as a normal hyperbolic point without the need of any extra parameter.

Let $z^{\prime}=h(z, \varepsilon)$ be a 1-parameter family with $h$ a smooth function and $h(0, \varepsilon)=$ 0 , for $\varepsilon$ near zero. The family is called time-reversible if there exists a germ of a smooth involution $\phi: R^{n+m}, 0 \rightarrow R^{n+m}, 0$ satisfying the relation $h(\phi(z), \varepsilon)=$ $-\phi^{\prime}(z) h(z, \varepsilon)$.

We point out some properties of reversible vector fields:

(a) The phase portrait of $z^{\prime}=h(z, \varepsilon)$ is symmetric with respect to Fix $(\phi)=$ $\left\{(x, y) \in R^{n+m} \mid \phi(x, y)=(x, y)\right\}$.

(b) If $\gamma(t)$ is a solution of $z^{\prime}=h(z, \varepsilon)$, then so is $\phi(\gamma(-t))$.

(c) Any orbit meeting Fix $(\phi)$ at two different points is a periodic orbit. In this case it is called a symmetric periodic orbit.

(d) Any singular point or periodic orbit on $\operatorname{Fix}(\phi)$ cannot be an attractor or a repeller.

(e) Intersection of (un)-stable manifolds with fixed sets of $\phi$ imply the existence of heteroclinic or homoclinic orbits.

Definition 1.3. The system $z^{\prime}=h(z, \varepsilon)$ is said to be time-reversible of type $(n+m ; k)$, or simply $(n+m ; k)$-reversible, if the dimension of the fixed point set of $\phi, F i x(\phi)$, is equal to $k$.

It should be noted that in our context the linearity of a reversing involution is not relevant. Reversible dynamical systems are a well-established mathematical subject widely discussed in the physics literature (see [5, 8] for further details, a historical review and related topics). 
Our main motivation is that many phenomena in physics and engineering involving reversible singular perturbation problem appear naturally.

Example 1.4 (see [4]). Imagine a particle with unit mass moving along a line under the influence of a periodic potential $\alpha\left(1-\cos y_{1}\right)$ and fixed external force $F$ such that the particle has coordinate $y_{1}$, momentum $y_{2}$ and its time-averaged kinetic energy is kept constant via a feedback mechanism involving a friction coefficient $x$. The equations of motion are

$$
\left\{\begin{array}{l}
\varepsilon \dot{x}=y_{2}^{2}-1 \\
\dot{y}_{1}=y_{2} \\
\dot{y_{2}}=F-\alpha \sin \left(y_{1}\right)-x y_{2},
\end{array}\right.
$$

where the parameter $\varepsilon>0$ is the thermostat strength. For $\varepsilon$ near zero, this system is singular and time-reversible with respect to the involution $\phi\left(x, y_{1}, y_{2}\right)=$ $\left(-x, y_{1},-y_{2}\right)$.

Let $X_{\varepsilon}$ be a reversible singularly perturbed vector field and let $\Gamma$ be a singular orbit. A rough description of the main results of the paper are as follows:

(1) Assume that $X_{\varepsilon}$ is of type $(k ; k-1)$. We give conditions on $X_{\varepsilon}$ under which $\Gamma$ is approximated by regular orbits (see Theorem A). In addition we exhibit conditions, for $k=2$ (resp. $k \geq 2$ ), under which $X_{\varepsilon}$ possesses a periodic orbit (resp. heteroclinic cycle) $\Gamma_{\varepsilon}$ converging to $\Gamma$. In section 2 we present a brief survey of the techniques which lead to a geometric analysis of singularly perturbed problems. In section 3 we prove Theorem A and discuss the existence of infinitely many periodic orbits or heteroclinic cycles converging to singular orbits.

(2) We present conditions for a $(1+m, k)$-reversible singularly perturbed vector field $X_{\varepsilon}$, where $k<m$, having a singular orbit $\Gamma$, to be approximated by a vector field $Y_{\varepsilon}$, which has periodic orbits (resp. heteroclinic cycles) $\Gamma_{\varepsilon}$, when $m=1$ (resp. $m \geq 1$ ), such that $\Gamma_{\varepsilon} \rightarrow \Gamma$, as $\varepsilon \downarrow 0$ (see Theorem B). Section 4 is devoted to prove Theorem B.

\section{BASIC FACTS OF GSP-THEORY}

The foundation of "GSP-theory", which is briefly summarized here, was laid by Fenichel in [2].

Consider the slow manifold of system (1.1), $\mathcal{S}$, which is given implicitly by $f(x, y, 0)=0$.

Definition 2.1. We say that $\left(x_{0}, y_{0}\right) \in \mathcal{S}$ is normally hyperbolic if the real parts of the eigenvalues of $D_{1} f\left(x_{0}, y_{0}, 0\right)$ are nonzero.

Consider $K \subset \mathcal{S}$ a compact set such that every $p \in K$ is normally hyperbolic. Assume that $D_{1} f(p, 0)$ has $k^{s}$ eigenvalues with negative real parts and $k^{u}$ eigenvalues with positive real parts. There are locally invariant manifolds, $\mathcal{C}^{s}, \mathcal{C}$ and $\mathcal{C}^{u}$, containing $K \times\{0\}$ and tangent to the corresponding center-stable, center and center-unstable eigenspaces of the linearization supplemented by the equation $\varepsilon^{\prime}=0$.

Theorem 2.2 (Fenichel [2]). If $\mathcal{N} \subset K$ is a $j$-dimensional invariant manifold of the slow system with $a\left(j+j^{s}\right)$-dimensional local stable manifold $W^{s}$ and $a\left(j+j^{u}\right)$ dimensional local unstable manifold $W^{u}$, then there exists an $\varepsilon$-continuous family 
$\mathcal{N}_{\varepsilon}$ such that

a) $\mathcal{N}_{0}=\mathcal{N}$, and

b) $\mathcal{N}_{\varepsilon}$ is an invariant manifold of $X_{\varepsilon}$ with a $\left(j+j^{s}+k^{s}\right)$-dimensional local stable manifold $\mathcal{N}_{\varepsilon}^{s}$ and $a\left(j+j^{u}+k^{u}\right)$-dimensional local unstable manifold $\mathcal{N}_{\varepsilon}^{u}$.

The importance of this theorem is that every structure of the slow system which persists under regular perturbation persists under singular perturbation by restriction of the flow of the fast system to the center manifold.

The next step is to decide if a singular orbit can be approached by regular orbits.

Suppose that $\mathcal{F}^{s}$ and $\mathcal{F}^{u}$ are families of stable and unstable manifolds that foliate $\mathcal{C}^{s}$ and $\mathcal{C}^{u}$, respectively.

Definition 2.3. Submanifolds $M_{1}$ and $M_{2}$ of a manifold $M$ intersect transversally (denoted $M_{1} \pitchfork M_{2}$ ) at a point $p \in M_{1} \cap M_{2}$ if $T_{p} M=T_{p} M_{1}+T_{p} M_{2}$.

Theorem $2.4([3])$. If $W_{1}^{u}\left(\mathcal{N}_{1}\right)$ and $W_{2}^{s}\left(\mathcal{N}_{2}\right)$ are on $K$ and

$$
\left(\mathcal{N}_{1}^{u}=\bigcup_{p \in W_{1}^{u}} \mathcal{F}^{u}(p)\right) \pitchfork\left(\mathcal{N}_{2}^{s}=\bigcup_{p \in W_{2}^{s}} \mathcal{F}^{s}(p)\right),
$$

then there exists an orbit of $X_{\varepsilon}$ connecting $\mathcal{N}_{1, \varepsilon}$ and $\mathcal{N}_{2, \varepsilon}$.

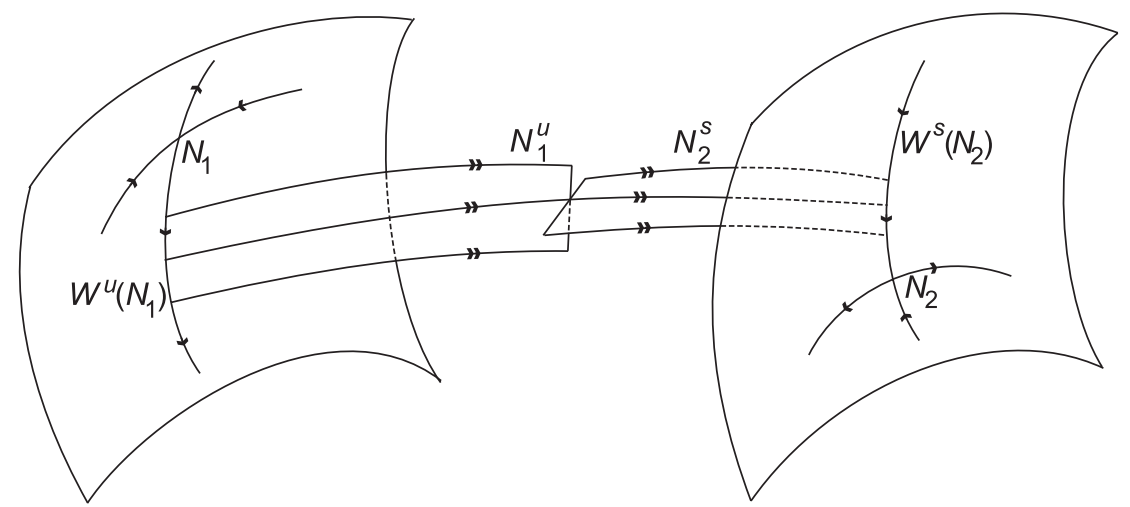

FiguRE 1. Transversal intersection of invariant manifolds.

We emphasize that Theorem 2.4 was obtained under the normal hyperbolicty hypothesis.

Dumortier and Roussarie presented a method based on the blowup techniques that leads to a rigorous geometric analysis of the nonnormally hyperbolic case [1. The idea is to saturate the flow and blow up the singularity so that after desingularization the orbits, for $\varepsilon$ near zero, are obtained by transverse intersection of center manifolds associated to the lines of normally hyperbolic singularities. Their main example is the singular perturbation problem

$$
X_{\varepsilon, a}(x, y)=\left(y-\frac{x^{2}}{2}-\frac{x^{3}}{3}, \varepsilon(a-x)\right) .
$$




\section{Singularly Perturbed ReVersible VeCtor Fields \\ OF TYPE $(n+m ; n+m-1)$}

Here we examine the solutions of reversible singular perturbation problems expressed by $X_{\epsilon}$ as in (1.1).

Definition 3.1. We say that $X_{\epsilon}$ satisfies the QG-condition if

(1) $(x, y) \in R^{n+m}, \varepsilon \geq 0$, and $f, g \in C^{\infty}$.

(2) The slow manifold $\mathcal{S}=\{(x, y) \mid f(x, y, 0)=0\}$ is a $k$-manifold with $k \leq$ $n+m-1$.

(3) $p \in \mathcal{S} \pitchfork \operatorname{Fix}(\phi)$ is a nonnormally hyperbolic point.

(4) $\mathcal{N}_{1}$ and $\mathcal{N}_{2}$ are $\phi$-symmetric invariant manifolds on $\mathcal{S}$ and $\Gamma$ is a singular orbit passing through $p$ that is composed of 3 pieces: an orbit of the reduced problem in the unstable manifold $W_{1}^{u}\left(\mathcal{N}_{1}\right)$, an orbit of the reduced problem in the stable manifold $W_{2}^{s}\left(\mathcal{N}_{2}\right)$, and a heteroclinic orbit of the fast problem that connects them.

(5) $p$ is the unique nonnormally hyperbolic point on $\Gamma$.

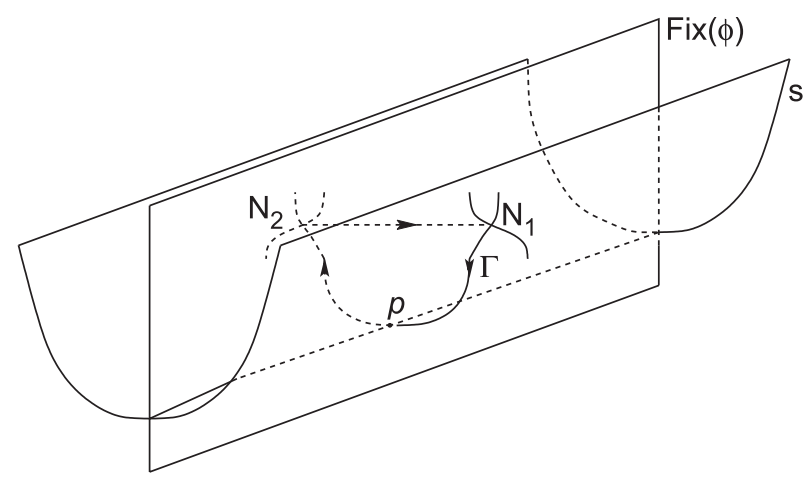

FiguRE 2. QG-condition

Theorem A. Suppose that $X_{\varepsilon}$ given by (1.1) satisfies the $Q G$-condition. There exists a neighborhood $U \subset R^{n+m}$ of $p$ as in (3), such that if $\Gamma \subset U$, then for each $\varepsilon>0$ there exists an orbit $\Gamma_{\varepsilon}$ of $X_{\varepsilon}$ that approaches $\Gamma$ as $\varepsilon \downarrow 0$, with respect to the Hausdorff distance.

Proof. Consider the auxiliary vector field $X_{\varepsilon}^{*}(x, y, \varepsilon)=(f(x, y, \varepsilon), \varepsilon g(x, y, \varepsilon), 0)$ on $R^{n+m+1}$. Without lost of generality, suppose that the involution $\phi$ is linear and $F i x(\phi)=\left\{(x, y): x_{1}=0\right\}$. Take $q \neq p$ on $\Gamma \cap F i x(\phi)$. Such a point exists because $\Gamma$ connects $\phi$-symmetric invariant manifolds on $\mathcal{S}$. Let $l=\{(x, y, \varepsilon):(x, y)=$ $\left.q, 0 \leq \varepsilon<\varepsilon_{0}\right\}$, where $\varepsilon_{0}$ is some sufficiently small positive number. Thus $l$ is a segment of a curve transverse to Fix $(\phi)$ at $q$. Denote by $C_{l}$ the saturate of $l$, that is, the closure of the union of segments of orbits of $X_{\varepsilon}^{*}$ through the points of $l$ and taken between the first intersection of this curve with $F i x(\phi)$ in negative time $\left(C_{l}^{-}\right)$and in positive time $\left(C_{l}^{+}\right)$. The orbits on $C_{l}$ are the orbits of $X_{\varepsilon} . C_{l}$ crosses Fix $(\phi)$ transversally because the singular orbit $\Gamma$ does. Finally, the reversibility of $X_{\varepsilon}$ implies that $C_{l}^{-} \cap$ Fix $(\phi)=C_{l}^{+} \cap$ Fix $(\phi)$. To complete the proof it is enough to choose $\Gamma_{\varepsilon}$ to be an orbit contained on $C_{l}$. 


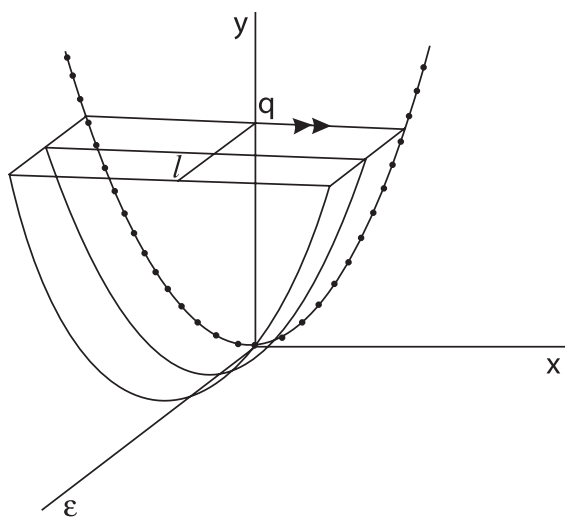

Figure 3. Center manifold $C_{l}$

Corollary 3.2. If $\mathcal{N}_{1}$ and $\mathcal{N}_{2}$, as in the $Q G$-condition, are hyperbolic singular points of the slow system, then the family obtained in Theorem A is composed of heteroclinic cycles.

Proof. It is enough to combine Theorem A and Theorem 2.2.

Example 3.3. Consider the family of reversible vector fields on $R^{3}$ given by $X_{\varepsilon}\left(x, y_{1}, y_{2}\right)=\left(y_{2}-x^{2}, \varepsilon y_{1} x, \varepsilon y_{1} x\right)$ and the linear involution on $R^{3}$ given by $\phi\left(x, y_{1}, y_{2}\right)=\left(-x, y_{1}, y_{2}\right)$. The family $X_{\varepsilon}$ satisfies $X_{\varepsilon} \circ \phi=-\phi \circ X_{\varepsilon}$. So it is $\phi$-reversible and the phase portrait of $X_{\varepsilon}$ is symmetric with respect to Fix $(\phi)=$ $\left\{\left(0, y_{1}, y_{2}\right) \mid y_{1}, y_{2} \in R\right\}$. The reduced system is

$$
\left\{\begin{array}{l}
y_{2}=x^{2}, \\
y_{1}^{\prime}=y_{1} x, \\
y_{2}^{\prime}=y_{1} x,
\end{array}\right.
$$

and the projection of some trajectories of the slow manifold on the $x y_{1}-$ plane is composed of parabolas with vertices on $\overrightarrow{0 y_{1}}$. All points in $\overrightarrow{0 x}$ are singularities. Consider a singular orbit $\mathcal{N}$ such that its projection on the $x y_{1}$-plane is a parabola with vertex $\left(0, y_{0}\right)$, and $y_{0}<0$. For $\varepsilon$ sufficiently small, we may select an orbit $\Gamma_{\varepsilon}$ of $X_{\varepsilon}$ that approaches the singular orbit $\Gamma$ with respect to the Hausdorff distance.

This situation is generic. In our terminology it satisfies $\frac{\partial f}{\partial x}(0,0)=0, \frac{\partial^{2} f}{\partial x^{2}}(0,0) \neq$ 0 and $\left(\frac{\partial f}{\partial y_{1}}(0,0)\right)^{2}+\left(\frac{\partial f}{\partial y_{2}}(0,0)\right)^{2} \neq 0$.

The next theorem states the existence of a one-parameter family of periodic solutions converging to the singular orbit in the $(2 ; 1)$-reversible case.

Theorem 3.4. Suppose the system $X_{\varepsilon}$, given by (1.1), satisfies the $Q G$-condition, is $\phi$-reversible with $\phi(x, y)=(-x, y)$ and satisfies $\frac{\partial f}{\partial y}(0,0,0) \cdot \frac{\partial g}{\partial x}(0,0,0)<0$. If $\Gamma$ is a singular orbit of $X_{0}$ passing through $(0,0)$ and connecting $\phi$-symmetric points on the slow manifold, then there exists a sequence of periodic orbits $\Gamma_{\varepsilon}$, of $X_{\varepsilon}$, that approaches $\Gamma$ for $\varepsilon \downarrow 0$.

Proof. If $X_{\varepsilon}$ is $\phi$-reversible, then we have $f(x, y, \varepsilon)=\varphi\left(x^{2}, y, \varepsilon\right)$ and $g(x, y, \varepsilon)=$ $x \psi\left(x^{2}, y, \varepsilon\right)$ for some smooth functions $\varphi$ and $\psi$ on $R^{2}$. The singular points for $\varepsilon>0$ 
are given by the equations $x \psi\left(x^{2}, y, \varepsilon\right)=0$ and $\varphi\left(x^{2}, y, \varepsilon\right)=0$. By the implicit function theorem there is a smooth function $y=y(\varepsilon)$ provided that $\frac{\partial f}{\partial y}(0,0,0) \neq 0$. Thus we have a curve $(0, y(\varepsilon), \varepsilon)$ composed of singular points of $X_{\varepsilon}$. Moreover $\frac{\partial f}{\partial x}(0, y(\varepsilon), \varepsilon)=\frac{\partial g}{\partial y}(0, y(\varepsilon), \varepsilon)=0 ; \frac{\partial f}{\partial y}(0, y(\varepsilon), \varepsilon) \cdot \frac{\partial g}{\partial x}(0, y(\varepsilon), \varepsilon)<0$ and $\frac{\partial f}{\partial y}$ and $\frac{\partial g}{\partial x}$ are continuous functions. Finally, the reversibility condition ensures that the singularity $(0, y(\varepsilon), \varepsilon)$ is a center for $X_{\varepsilon}$.

\section{Singularly perturbed ReVersible Vector Fields OF TYPE $(n+m ; k)$}

In this section we treat the singular perturbation problem for $(n+m ; k)$ reversible vector fields with $k<n+m-1$.

Consider $X_{\varepsilon, a}$ a 2-parameter family of reversible vector fields on $R^{3}$ that is of type $(1+2 ; 1)$ with respect to the involution $\phi\left(x, y_{1}, y_{2}\right)=\left(-x,-y_{1}, y_{2}\right)$, given by $X_{\varepsilon, a}\left(x, y_{1}, y_{2}\right)=\left(f\left(x, y_{1}, y_{2}\right), \varepsilon g_{1}\left(x, y_{1}, y_{2}, a\right), \varepsilon g_{2}\left(x, y_{1}, y_{2}, a\right)\right)$.

Let $\mathcal{N}_{1}$ and $\mathcal{N}_{2}$ be $\phi$-symmetric invariant manifolds on the slow manifold $\mathcal{S}$ and let $\Gamma \subset S$ be a singular orbit passing through 0 , which is the unique nonnormally hyperbolic point on $\Gamma$. Assume that $\Gamma$ is composed of 3 pieces $\Gamma_{1}, \Gamma_{2}$ and $\Gamma_{3}$. Such curves are characterized by:

(1) $\Gamma_{1}$ is an orbit of the reduced problem in the unstable manifold $W_{1}^{u}\left(\mathcal{N}_{1}\right)$.

(2) $\Gamma_{2}$ is an orbit of the reduced problem in the stable manifold $W_{1}^{s}\left(\mathcal{N}_{2}\right)$.

(3) $\Gamma_{3}$ is an orbit of the fast problem connecting $\Gamma_{1}$ and $\Gamma_{2}$.

Take $p \in \Gamma \cap\left\{\left(0,0, y_{2}\right) \mid y_{2} \in R\right\}$ and $l$ a segment that is transversal to the line $\left\{\left(0,0, y_{2}\right) \mid y_{2} \in R\right\}$ on $p$. Let $\gamma_{\varepsilon, a}(p)$ be the orbit of $X_{\varepsilon, a}$ passing thought $p \in l$. There is a sequence of regular orbits $\Gamma_{\varepsilon, a}$ approaching $\Gamma$ when $\varepsilon \downarrow 0$, provided there exists a curve $a=a(\varepsilon)$ on the parameter space $(\varepsilon, a)$ such that for the parameter values on this curve, $\gamma_{\varepsilon, a}(p)^{+}=\gamma_{\varepsilon, a}(p)^{-}$.

Another way to approach the singular orbit is when there is a curve $a=a(\varepsilon)$ such that for $\varepsilon>0, X_{\varepsilon, a(\varepsilon)}$ is of type $(1+2 ; 2)$ with respect to an involution $\widetilde{\phi}$. In fact, in this case the center manifold crosses the set of fixed points of $\widetilde{\phi}$ and so we get the desired regular orbits.

Example 4.1. Consider $\phi(x, y)=\left(-x,-y_{1}, y_{2}\right), \widetilde{\phi}(x, y)=(-x, y)$ and $X_{\varepsilon, a}(x, y)$ $=\left(-y_{2}+x^{2},-\varepsilon x^{2} y_{1}^{2}+a x^{2} y_{1}^{2}, \varepsilon x y_{2}\right)$. If $a=0$, then $X_{\varepsilon, 0}$ is of the type $(1+2 ; 1)$ with respect to the involution $\phi$ and it is not of the type $(1+2 ; 2)$ with respect to the involution $\widetilde{\phi}$. But if $a=\varepsilon$, then $X_{\varepsilon, \varepsilon}$ is of type $(1+2 ; 2)$ with respect to the involution $\widetilde{\phi}$. We denote $Y_{\varepsilon}=X_{\varepsilon, \varepsilon}$ and consider $\Gamma$ an orbit of the reduced problem associated to

$$
Y_{0}:\left\{\begin{array}{l}
y_{2}=x^{2}, \\
y_{1}^{\prime}=0, \\
y_{2}^{\prime}=x y_{2} .
\end{array}\right.
$$

We choose $\Gamma$ such that $x^{\prime}=\frac{x^{2}}{2}, y_{1}=0$ and $y_{2}=x^{2} . X_{\varepsilon, \varepsilon}$ is reversible with involution $\widetilde{\phi}$ and thus its phase portrait is $y_{1} y_{2}$-symmetric. Then as $\varepsilon$ goes towards 0 , a regular orbit of $X_{\varepsilon, \varepsilon}$ approaches $\Gamma$.

Fundamental Lemma. Let $X_{\varepsilon}$ be a $(n+m ; k)$-reversible vector field with respect to a diagonal involution $\varphi$ (i.e., the matrix that represents $\varphi$ is diagonal), given by 


$$
\begin{aligned}
& X_{\varepsilon}(x, y)=(f(x, y, \varepsilon), \varepsilon g(x, y, \varepsilon)) \text {. Assume that } f \text { satisfies } \\
& \qquad \begin{array}{l}
f_{1}(x, y)=f_{1}\left(-x_{1}, x_{2}, \ldots, x_{n}, y\right), \\
f_{i}(x, y)=-f_{i}\left(-x_{1}, x_{2}, \ldots, x_{n}, y\right) \forall i \in\{1,2, \ldots, n\} .
\end{array}
\end{aligned}
$$

Then there exists a two-parameter family $X_{\varepsilon, a}$ that is $\varphi$-reversible for all $(\varepsilon, a)$ and $\varphi_{0}$-reversible if $a=\varepsilon$. Here $\varphi_{0}(x, y)=\left(-x_{1}, x_{2}, \ldots, x_{n}, y\right)$.

Proof. Let $\left\{i_{1}, i_{2}, \ldots, i_{s}\right\}$ be the indices of $x$ and let $\left\{j_{1}, j_{2}, \ldots, j_{r}\right\}$ be the indices of $y$, where the minus sign is given in the definition of $\varphi$. Obviously $s+r=$ $n+m-k$, because $\varphi$ is of type $(n+m ; k)$. For each $j \in\left\{j_{1}, j_{2}, \ldots, j_{r}\right\}$, because $X_{\varepsilon}$ is $\varphi$-reversible, we have that $g_{j}(x, y, \varepsilon)=g_{j}(\varphi(x, y), \varepsilon)$. This equation implies that the expansion in Taylor's series of $g_{j}$ has only monomials of type $x_{1}^{k_{1}} x_{2}^{k_{2}} \ldots x_{n}^{k_{n}} y_{1}^{l_{1}} y_{2}^{l_{2}} \ldots y_{m}^{l_{m}}$ such that $k_{i_{1}}+k_{i_{2}}+\cdots+k_{i_{s}}+l_{j_{1}}+l_{j_{2}}+\cdots+l_{j_{r}}$ is even. For each $j \in\{1,2, \ldots, m\} \backslash\left\{j_{1}, j_{2}, \ldots, j_{r}\right\}$, again using that $X_{\varepsilon}$ is $\varphi$-reversible, we have $g_{j}(x, y, \varepsilon)=-g_{j}(\varphi(x, y), \varepsilon)$. So, in this case we have that $g_{j}$ has only monomials $x_{1}^{k_{1}} x_{2}^{k_{2}} \ldots x_{n}^{k_{n}} y_{1}^{l_{1}} y_{2}^{l_{2}} \ldots y_{m}^{l_{m}}$ such that $k_{i_{1}}+k_{i_{2}}+\cdots+k_{i_{s}}+l_{j_{1}}+l_{j_{2}}+$ $\cdots+l_{j_{r}}$ is odd. Observe that $X_{\varepsilon}$ is also $\varphi_{0}$-reversible provided that each $g_{j}$ satisfies $g_{j}(x, y, \varepsilon)=-g_{j}\left(-x_{1}, x_{2}, \ldots, x_{n}, y, \varepsilon\right)$. The above equation is equivalent to saying that $g_{j}$ has only monomials $x_{1}^{k_{1}} x_{2}^{k_{2}} \ldots x_{n}^{k_{n}} y_{1}^{l_{1}} y_{2}^{l_{2}} \ldots y_{m}^{l_{m}}$ such that $k_{1}$ is odd. Consider $Y_{\varepsilon}(x, y)=(0, h(x, y, \varepsilon))$, where $h_{j}(x, y, \varepsilon)$ has all monomials of $g_{j}$ except the ones where $k_{1}$ is even. Now define $X_{\varepsilon, a}=X_{\varepsilon}-a Y_{\varepsilon}$. By construction $X_{\varepsilon, a}$ is $\varphi$-reversible for all $(\varepsilon, a)$ and $X_{\varepsilon, \varepsilon}$ is $\varphi_{0}$-reversible.

Corollary 4.2. Consider the reversible singular perturbation problem $X_{\varepsilon}(x, y)=$ $\left(f\left(x^{2}, y\right), \varepsilon g_{1}(x, y), \ldots, \varepsilon g_{m}(x),\right)$, which is $(1+m ; k)$-reversible with respect to the involution $\phi(x, y)=\left(-x,-y_{1}, \ldots,-y_{m-k}, y_{m+1-k}, \ldots, y_{m}\right)$. Then there exists a two-parameter family $X_{\varepsilon, a}$ such that $X_{\varepsilon, \varepsilon}$ is $(1+m ; m)$-reversible with respect to $\widetilde{\phi}(x, y)=(-x, y)$.

Let $K \subset R^{1+m}$ be a compact set and let $\mathfrak{X}_{1}=\left\{X=(f, g): K \subset R^{1+m} \rightarrow\right.$ $\left.R^{1+m} \mid f, g \in C^{1}\right\}$.

For $X, Y \in \dddot{X}_{1}$ we define $d(X, Y)=\sup _{p \in K}\{\|X(p)-Y(p)\|,\|D X(p)-D Y(p)\|\}$.

Theorem B. Let $X_{\varepsilon}(x, y)=\left(f\left(x^{2}, y\right), \varepsilon g_{1}(x, y), \ldots, \varepsilon g_{m}(x, y)\right)$ be reversible of type $(1+m, k)$ with respect to the involution

$$
\phi(x, y)=\left(-x,-y_{1}, \ldots,-y_{m-k}, y_{m+1-k}, \ldots y_{m}\right)
$$

and let $K \subset R^{1+m}$ be a compact set. Consider $\Gamma_{0} \subset K$ a singular orbit of $X_{0}$ passing through $(0,0) \in S \pitchfork$ Fix $(\phi)$ and connecting points $p, q$ on the slow manifold, that are symmetric with respect to $\phi$.

a) There exists $Y_{\varepsilon}: K \subset R^{1+m} \rightarrow R^{1+m},(1+m, m)$-reversible with respect to involution $\widetilde{\phi}(x, y)=(-x, y)$, such that $d\left(Y_{\varepsilon}, X_{\varepsilon}\right) \rightarrow 0$ as $\varepsilon \downarrow 0$. There exists $\Gamma_{\varepsilon}$ regular orbits of $Y_{\varepsilon}$ such that $\Gamma_{\varepsilon} \rightarrow \Gamma_{0}$ with respect to the Hausdorff distance.

b) If $m=1$ and $\frac{\partial f}{\partial y}(0,0) \cdot \frac{\partial g}{\partial x}(0,0)<0$, then there exists $\Gamma_{\varepsilon}$, a periodic orbit of $Y_{\varepsilon}$, such that $\Gamma_{\varepsilon} \rightarrow \Gamma_{0}$ with respect to the Hausdorff distance.

c) If $m \geq 1$ and $p, q$ are $\phi$-symmetric hyperbolic singular points of the slow system, then there exists a family of heteroclinic cycles of $Y_{\varepsilon}, \Gamma_{\varepsilon}$, such that $\Gamma_{\varepsilon} \rightarrow \Gamma_{0}$, as $\varepsilon \downarrow 0$, with respect to the Hausdorff distance. 
Proof. For a), it is enough to define $Y_{\varepsilon}=X_{\varepsilon, \varepsilon}$, where $X_{\varepsilon, a}$ is the family given in the corollary of the Fundamental Lemma.

Part b) follows from Theorem 3.4 by observing that if $Y_{\varepsilon}$ is near $X_{\varepsilon}$ in the $\mathcal{C}^{1}$-topology, then the the hypothesis on $X_{\varepsilon}$ still holds for $Y_{\varepsilon}$.

Part c) is the corollary of Theorem A. Again the convergence in the $\mathcal{C}^{1}$-topology implies that $\Gamma_{0}$ satisfies the QG-condition. If $(0,0) \in S \pitchfork F i x(\phi)$, then $(0,0) \in S \pitchfork$ Fix $(\widetilde{\phi})$ since $\frac{\partial f}{\partial x}(0,0)=0$ (nonnormally hyperbolic). So $\Gamma_{0}$ is tangent to the $x$-axis and the $x$-axis is not contained in Fix $(\widetilde{\phi})$.

Consider the following one-parameter family of vector fields in $R^{3}$ :

$$
X_{\lambda}:\left\{\begin{array}{l}
\varepsilon \dot{x}=y_{2}^{2}-1+\lambda y_{1}, \\
\dot{y_{1}}=y_{2}, \\
\dot{y_{2}}=F-\alpha \sin \left(y_{1}\right)-x y_{2} .
\end{array}\right.
$$

Observe that the unperturbed system $X_{0}$, is Example 1.4. With this picture in mind we can give a crude qualitative description of the perturbed system, in the sense that for small $\lambda \neq 0 \quad X_{\lambda}$ satisfies the hypotheses of Theorem B.

\section{REFERENCES}

[1] F. Dumortier, R. Roussarie. Canard Cycles and Center Manifolds, Memoirs of the A.M.S, V. 121, 1996. MR1327208 (96k:34113)

[2] N. Fenichel. Geometric Singular Perturbation Theory for Ordinary Differential Equations, J.D.E 31, 53-98, 1979. MR0524817 (80m:58032)

[3] P. Szmolyan. Transversal Heteroclinic and Homoclinic Orbits in Singular Perturbation Problems , J.D.E 92, 252-281, 1991. MR1120905 (92e:58185)

[4] W. G. Hoover, H. A. Posch, B. L. Holian, M. J. Gillan, M. Mareshal and C. Massobrio. Dissipative irreversibility from Nosé's reversible mechanics, Mol. Simulation 1, 79-86, 1987.

[5] J. S. W. Lamb and J. A. G. Roberts. Time-reversal symmetry in dynamical systems: a survey. Phys. D, 112(1-2):1-39, 1998. Time-reversal symmetry in dynamical systems (Coventry, 1996). MR:1605826 (99b:58174)

[6] M. Krupa and P. Szmolyan. Extending geometric singular perturbation theory to nonhyperbolic points: fold and Canard points in two dimensions, SIAM J. Math. Anal. 33, 2, 286-314, 2001. MR 1857972 (2002g:34117)

[7] M. Krupa and P. Szmolyan. Extending slow manifolds near transcritical and pitchfork singularities, Nonlinearity 14, 1473-1491, 2001. MR1867088(2002k:34112)

[8] J. A. G. Roberts and G. R. W. Quispel. Chaos and time-reversal symmetry. Order and chaos in reversible dynamical systems. Phys. Rep., 216(2-3):63-177, 1992. MR1173588 (94a:58135)

ibilce, Universidade Estadual Paulista, São José do Rio Preto, SP, CEP 15054-000, BRAZIL

E-mail address: buzzi@mat.ibilce.unesp.br

ibilce, Universidade Estadual Paulista, São José do Rio Preto, SP, CEP 15054-000, BRAZIL

E-mail address: prs@mat.ibilce.unesp.br

Instituto de Matemática, Estatística e Computação Cientificica, Universidade Estadual de Campinas, Campinas, SP, CEP 13081-970, Brazil

E-mail address: teixeira@ime.unicamp.br 